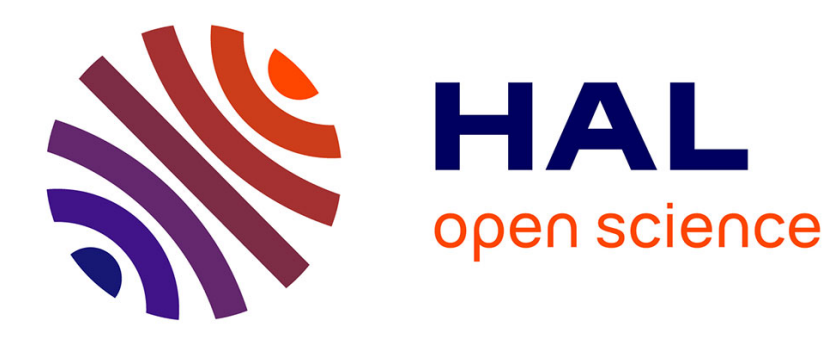

\title{
La fierté nationale de l'esclave [A propos de la récente vague nationaliste en Chine]
}

\author{
Liu Xiaobo, Frank Muyard
}

\section{To cite this version:}

Liu Xiaobo, Frank Muyard. La fierté nationale de l'esclave [A propos de la récente vague nationaliste en Chine]. Perspectives chinoises, 1997, 40, pp.34-37. 10.3406/perch.1997.2120 . halshs-02515773

\section{HAL Id: halshs-02515773 \\ https://shs.hal.science/halshs-02515773}

Submitted on 23 Mar 2020

HAL is a multi-disciplinary open access archive for the deposit and dissemination of scientific research documents, whether they are published or not. The documents may come from teaching and research institutions in France or abroad, or from public or private research centers.
L'archive ouverte pluridisciplinaire HAL, est destinée au dépôt et à la diffusion de documents scientifiques de niveau recherche, publiés ou non, émanant des établissements d'enseignement et de recherche français ou étrangers, des laboratoires publics ou privés. 


\section{La fierté nationale de l'esclave [A propos de la récente vague} nationaliste en Chine]

A propos de la récente vague nationaliste en Chine Liu Xiaobo, Frank Muyard

\section{Citer ce document / Cite this document :}

Xiaobo Liu, Muyard Frank. La fierté nationale de l'esclave [A propos de la récente vague nationaliste en Chine]. In: Perspectives chinoises, ${ }^{\circ} 40,1997$. pp. 34-37;

doi : https://doi.org/10.3406/perch.1997.2120

https://www.persee.fr/doc/perch_1021-9013_1997_num_40_1_2120

Fichier pdf généré le 24/04/2018 
rité d'un handicapé moteur qui se moquerait d'un tétraplégique. Quand je me suis réellement trouvé dans un monde ouvert, j'ai en effet réalisé tout à coup que je n'étais ni un théoricien ni une grande figure, mais un homme ordinaire qui doit repartir de zéro.

En Chine, un arrière-plan d'ignorance me faisait apparaître plein de sagesse, la couardise des incompétents donnait du relief à mon courage, et l'idiotie congénitale me faisait paraître par contraste en bonne santé. Mais aux Etats-Unis, privé de ce contexte d'ignorance, je me suis retrouvé démuni de sagesse; une fois le mur de l'idiotie écroulé, je n'étais plus qu'un homme malade; une fois la faiblesse générale évacuée, je n'étais plus qu'un poltron qui n'ose pas se regarder en face. En Chine, je vivais sur une réputation injustifiée à quatrevingt dix pour cent. En Occident, pour la première fois j'ai fait face à la vie réelle et aux durs choix de l'exis- tence. C'est quand on chute d'un coup du haut de ses illusions, que l'on réalise n'avoir jamais gravi de sommets et n'avoir toujours fait que se débattre dans un trou. Ma femme, Tao Li, m'écrivit un jour : "Xiaobo, en apparence, tu es le fils rebelle de cette société, mais en réalité, tu partages avec elle une identité profonde. Cette société peut, tout en s'opposant à toi, te tolérer, te pardonner, t'inspirer, et même t'encourager. Tu es comme un motif ornemental au verso de cette société". A l'époque, je n'avais pas compris ces mots, mais maintenant, quand j'y repense, ils touchaient droit au but. Je remercie $\mathrm{Tao} \mathrm{Li}$, elle n'est pas seulement ma femme, mais encore la critique la plus acérée, devant laquelle je m'incline.

Je ne peux plus reculer. Soit je franchis le précipice, soit je me brise les os. Quand on pense la réalité, on se doit d'être sur le champ de bataille.

\title{
La fierté nationale de l'esclave
}

\section{A propos de la récente vague nationaliste en Chine}

\author{
LIU XIAOBO (5) \\ TRADUIT PAR FRANK MUYARD
}

$\mathrm{P}$ EUT-ÊTRE est-ce la peur du sang et de la prison qui a submergé la conscience, peut-être est-ce la douloureuse leçon qui a éveillé la raison, peut-être est-ce l'esprit de profit qui a mis en déroute l'abstraction de la démocratie libérale, ou peut-être est-ce l'échec de la candidature de la Chine aux Jeux Olympiques qui a excité la fierté nationale. Mais, depuis les événements de Tian'anmen du 4 juin 1989 et surtout depuis le "voyage dans le sud" de Deng Xiaoping en 1992, toute la Chine, des cercles gouvernementaux aux milieux populaires, connaît un raz-de-marée de nationalisme et de retour au terroir. Sa rapidité, sa fureur, et sa longueur sont sans compararaison avec les précédents mouvements patriotiques initiés par le gouvernement. Comme si les plaies causées par les humiliations nationales des cent dernières années se rouvraient une fois encore.

\section{La grande chorale nationaliste}

Dans le "Programme pour une éducation patriotique" qu'il a établi, le gouvernement a mobilisé tous les moyens pour promouvoir la fierté nationale. Il n'épargne aucun effort pour organiser soirées, conférences, concerts, lectures, festivals, expositions et autres réunions dont le but est de propager la culture nationale. La télévision diffuse chaque jour un film patriotique. Des séries de livres sur la culture traditionnelle chinoise sortent à grands frais des maisons d'édition. La cérémonie du lever de drapeau sur la place Tian'anmen suscite quotidiennement l'attention des gens. Et les écoles primaires et secondaires doivent, elles aussi, selon un réglement en vigueur, procéder au lever du drapeau. Le gouvernement se sert également du nationalisme pour affronter la scène internationale, en particulier sur la question des droits de l'homme, où face aux pressions de la communauté internationale, il argue, tout en jouant de l'énorme marché chinois, des différences entre situations nationales, traditions et critères en matière de droits de l'homme, pour justifier le fait qu'il les écrase et dénoncer l'hégémonisme occidental.

Dans la population, la diffusion en direct à la télévision 
du rejet de la candidature chinoise à l'organisation des Jeux Olympiques a soulevé la tristesse et la colère de millions ct $\mathrm{dc}$ millions $\mathrm{dc}$ Chinois, qui attribuent cet échec aux Occidentaux et surtout aux Américains. Par la suite, Sunzi, Laozi, Yu le Grand, l'empereur Gaozu des Han, Xuanzong des Tang, l'impératrice $\mathrm{Wu}$ Zetian, les empereurs Taizong des Song, Kubilaï des Yuan, Taizu des Ming, Kangxi des Qing, ainsi que toute une série de concubines sont apparus les uns après les autres sur les écrans de télévision avec un éclat sans pareil. Les livres sur le YiJing, la lectures des hexa-

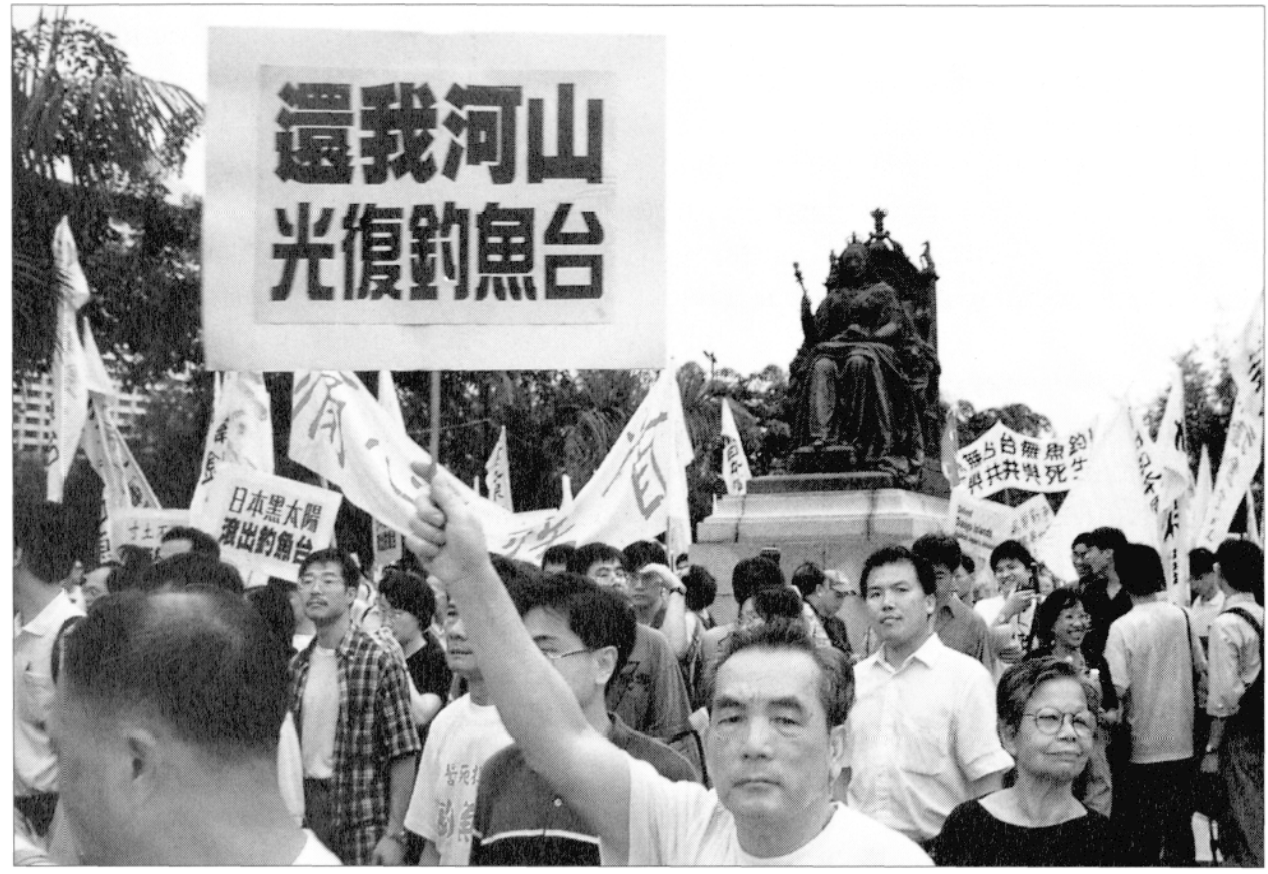

La question des Diaoyu tai a réveillé le nationalisme chinois grammes, la théorie du Qigong, la stratégie de Sunzi, les biographies des personnages de l'antiquité, etc. se sont répandus dans tous les kiosques du pays. La Vitrine de la civilisation chinoise, Villages de Chine, le Musée de cire de l'Antiquité, le Palais du Pélerinage vers l'Ouest, le Palais des Trois Royaumes et autres lieux de divertissements ont fleuri partout. Au-dessus des nouvelles constructions sont apparus de plus en plus de toits recourbés de style traditionnel. Liu Huifang, le personnage féminin principal de la série télévisée Espérances (Kewang), a été reconnue avant tout pour ses vertus traditionnelles (6). L'esprit décadent des lettrés traditionnels, avides d'épouses et de concubines, tel qu'il est décrit dans La Capitale déchue (Feidu) (7) a suscité un engouement national. Les films qui ont pour thème des histoires et des personnages traditionnels remportent encore des prix à la pelle.

Ceux qui ont séjourné à l'étranger n'ont pas non plus perdu de temps pour se joindre à la grande chorale nationaliste. Les années précédentes c'était la mode du départ à l'étranger, ces dernières années c'est la vague du retour, au point que même certains exilés du 4 Juin sont revenus discrètement en Chine. Ceux qui sont de retour expriment pour la plupart une grande frustration envers la vie à l'étranger, et la littérature et les films sur l'"envoi à l'étranger" (yang chadui) a dicté la mode du moment (8). Ce fut d'abord la vogue des romans-vérité comme "Une Chinoise de Manhattan" et "Un Pékinois à NewYork", puis l'explosion de la série-télévisée du même nom. Ces oeuvres présentent en gros les difficultés des Chinois à l'étranger, leur orgueil national exclusif, en même temps qu'ils dénoncent l'insensibilité du monde capitaliste. Peu importe qu'elles n'aient aucun rapport avec la vie réelle aux Etats-Unis : plus les mensonges sont gros, plus ils ont d'attrait, et leur succès est révélateur de l'état d'esprit des Chinois.

\section{Le rejet de l'Occident par les élites et le gouvernement}

L'humeur nationaliste manifestée par les élites académiques n'a jamais été aussi forte depuis le début de l'ouverture en 1978. Sans se fonder aussi directement sur des besoins politiques que le patriotisme gouvernemental ni être aussi émotionnel que celui des masses, leur nationalisme est enveloppé minutieusement dans un langage savant qui se pare de vertu. Ces élites sont insatisfaites de l'attitude de soumission envers l'Occident et de la tyrannie de la culture occidentale, comme si tout cela relevait directement de la conscience intellectuelle ou de la fierté nationale. Un certain nombre de jeunes professeurs influents de l'Université de Pékin prônent ainsi la régénération des études nationales (guoxue) et le retour à l'ère de Qianlong et de Jiaqing (9) dans le but de contrecarrer la mode académique du suivisme occidental. Quelques jeunes savants renommés font la promotion de la "sinité" dans de longs articles pour redresser l'esprit national en déclin. Les discussions qui se développent autour du "Choc des civilisations" de $S$. Huntington et de "L'Orientalisme" de E. Saïd concentrent leurs attaques sur la théorie du "centre occidental" et sur l'hégémonie de la culture européenne et américaine. Les écrivains de la "Nouvelle vague" qui se sont hissés sur la scène littéraire en imitant la littérature moderniste occidentale sont pour la plupart retournés à la "Terre jaune" (10). Bien qu'ils n'aient toujours pas le courage de regarder en face la réalité contemporaine chinoise et continuent d'estomper l'arrière-plan historique de leur oeuvres, leurs tendances au retour au terroir sau- 
tent aux yeux.

Plus dramatiquement, Zhang Yimou et Chen Kaige, qui ont ramené des Lions d'or et des Palmes de Cannes en faisant du terroir le thème privilégié de leur films, sont accusés par les théoriciens de faire du cinéma qui flatte le goût des étrangers. Et en plus on attribue à ce phénomène le nom savant très à la mode de "cinéma post-colonial".

Ce qui donne à réfléchir, c'est que les raisons avancées par le gouvernement et les élites intellectuelles pour rejeter l'Occident sont absolument les mêmes, et qu'ils utilisent les mêmes mots : hégémonisme de l'Occident, hégémonisne d'un système politique, hégémonisme d'une culture, réduisant en plus la formation de cet hégémonisme à une simple force matérielle - l'établissement d'une puissance économique et militaire sur la base du développement technique. Les Occidentaux exercent effectivement leur hégémonisme mondial en s'appuyant sur un capital économique et militaire. Mais les élites chinoises semblent oublier la liberté et la démocratie de l'Occident, et en reviennent à l'époque du "Mouvement d'occidentalisation" des Qing. C'est précisément à cette époque-là que les Occidentaux s'ouvrirent les portes de la Chine par la force des canonnières.

Le cycle de l'histoire va ainsi à une vitesse étonnante. Les tenants de l'occidentalisation qui appelaient à grands cris la liberté et la démocratie au moment du " 4 Juin" se sont transformés en un clin d'oeil en nationalistes opposés à l'hégémonie occidentale. Même $\mathrm{Su}$ Xiaokang, exilé aux Etats-Unis, a remis en question les dérives d"occidentalisation totale" et de "nihilisme national" présentes dans L'élégie du fleuve.

\section{Imiter l'étranger pour mieux le maîtriser}

Ce qui est regrettable, c'est que tous les outils avec lesquels ils s'opposent à l'hégémonie occidentale viennent de l'Occident, que les articles où ils défendent la culture chinoise sont bourrés de style et de termes conceptuels importés de l'Occident, et que leur "orientalisme" chéri avec lequel ils font la critique de l'ethnocentrisme occidental vient lui aussi des Etats-Unis. C'est vraiment une résurgence du mouvement "Imiter l'étranger pour mieux le maîtriser", qui a gagné le courant académique international pour défendre la dignité de la culture chinoise.

C'est en effet l'Occident qui domine la culture mondiale actuelle. Les Occidentaux déterminent les normes d'évaluation de toutes les productions culturelles mondiales, et ce n'est qu'en suivant leurs règles du jeu que l'on peut se faire reconnaître. Ceux qui se conforment aux normes occidentales sont reconnus comme dignes de l'humanité et de niveau international, sinon ils sont éliminés. Le prix Nobel de littérature pour les lettres, la Palme d'or, les Oscars et le Lion d'or pour le cinéma, la Biennale de Venise pour la peinture, le Registre des hommes célèbres d'Oxford et Cambridge pour les élites du monde entier, symbolisent la plus haute gloire inter- nationale et constituent les trésors convoités par tous les pays du monde. Les Chinois contemporains ont ainsi contracté le "complexe du Nobel" et le "complexe de l'Oscar" à un point pathologique. En revanche, les Orientaux n'ont pas de normes pour évaluer les Occidentaux, n'ont pas de règles du jeu reconnues du monde entier, et les productions culturelles occidentales n'ont ni besoin de remporter quelques prix en Orient, ni besoin d'aller au-devant des goûts orientaux.

En compensation à leur humilité et à leur auto-dénigrement face aux Occidentaux, les Orientaux ont ce plaisir de vanité étonnée que procure parfois l'obtention des faveurs du maître. Tagore obtient le prix Nobel, les Chinois ne peuvent que le présenter ainsi : "Le premier Asiatique à avoir obtenu le prix Nobel de littérature". Kawabata l'obtient à son tour, il est : "Le premier Japonais à avoir obtenu le prix Nobel de littérature". Mais ces dernières années, la formule "le premier" s'est transformée en "plus que zéro".

On voit donc qu'un prix étranger remporté par un Oriental est son laissez-passer pour le monde et lui donne une gloire éternelle qui, dans son propre pays, lui permet d'avoir la tête haute pour toujours. La docilité et la gentillesse des Orientaux devant les normes des Occidentaux est similaire à celles de ces enfants qui sortent à l'occasion une phrase de grande personne et reçoivent les éloges de leur parents. Pourtant, la formation de cette mentalité n'est pas seulement due aux normes occidentales. Ses causes en sont bien plus la personnalité d'esclave et le sentiment d'infériorité des Chinois. On a du mal à imaginer ce qu'il peut y avoir dans une personnalité d'esclave hors le complexe d'infériorité et la fierté tirée de son anomalie (la conscience du maitre).

\section{Les valeurs universelles, sources de la supériorité occidentale}

En allant encore un peu plus loin, le problème est : pourquoi cette inégalité mondiale ? Est-ce seulement parce que les Occidentaux sont riches et militairement puissants ? Apparemment non, puisque Kawabata et Kurosawa, dont le pays ne le cède en rien à l'Occident dans ces domaines, ne furent universellement reconnus comme des maîtres de niveau mondial qu'après avoir obtenu le Nobel et l'Oscar. Dans la formation de cette structure culturelle mondiale centrée sur l'Occident, l'argent et la force sont seulement des causes apparentes. Les raisons profondes sont, au contraire, les valeurs avancées des Occidentaux, et particulièrement celles élaborées depuis la Renaissance et qui s'attachent aux droits de l'homme, à la liberté, à l'égalité et à la dignité, ainsi que les structures politiques démocratiques bâties sur ces valeurs. Celles-ci sont bien plus conformes à la nature générale de l'humanité et bien plus favorables au développement et à la liberté humaine, elles constituent déjà des règles universellement admises auxquelles personne n'ose s'opposer ouvertement. Même les dictateurs 
orientaux doivent prôner la démocratie, même les élites intellectuelles insatisfaites de l'hégémonie occidentale doivent reconnaître les valeurs de démocratie, d'égalité, de liberté, de droits de l'homme et de dignité individuelle, même les promoteurs du néo-confucianisme, Du Weiming, Feng Youlan et Li Zehou doivent s'identifier à la démocratie et à la liberté pour faire la réclame de leur maxime "Régner à l'extérieur par le retour à la sagesse intérieure".

L'indignation constante des Chinois envers l'Occident, qui éclate si facilement dans des mouvements de masse patriotiques, a sa source à la fois dans l'entichement de la gloire passée, les lamentations sur le retard actuel, la peur de succomber aux tentations du monde ainsi qu'un sentiment d'infériorité et de faiblesse. Des épanchements irrationnels peuvent apaiser les humiliations des cent dernières années, mais ce n'est pas la haine raciale qui remédiera aux inégalités du monde. Mais plus ces épanchements seront aveugles, plus la haine sera entretenue, moins on pourra se laver de ces humiliations. On n'en a pas encore assez du cercle vicieux de l'histoire moderne chinoise ? Le souci morbide de la "face" du gouvernement des Qing a apporté les canons occidentaux, la furie des Boxers a amené des concessions encore plus inégales, la politique autarcique de Mao Zedong n'a pu durer que vingt ans, mais encore elle n'a maintenu l'illusion d'une fierté nationale qu'au prix de la mise en friche de l'économie, de l'abandon de la culture et de la perte totale de la dignité individuelle de chaque Chinois.

C'est pourquoi la première chose que les Chinois doivent faire, c'est, sur leur propre sol, recouvrer et mainte- nir la dignité qui fait de chacun un être humain, et faire face à la puissance de l'Occident avec une saine mentalité d'égalité. Alors seulement sera-t-il possible d'asseoir une authentique dignité nationale et individuelle. Le maître et l'esclave ne sont que les deux faces d'une même pièce. Je ne crois pas qu'un homme qui a l'habitude d'être un esclave chez lui puisse, une fois dehors, être fier de soi. Si notre rejet de l'hégémonie occidentale en arrivait au point où nous ne voudrions même plus de la liberté démocratique, nous serions alors éternellement dignes de n'être que des esclaves sans fierté.

1. Cf. Liu Xiaobo, Beiju. Shenmei. Ziyou (Tragédie, Esthétique, Liberté), Taipei, Fengyun shidai, 1989, et Xuanze de pipan (Critique sélective), Taipei, Fengyun shidai, 1989. On trouvera une bonne biographie critique de Liu Xiaobo dans Gu Xin, Zhongguo fanchuantongzhuyi de pinkun (La misère de l'anti-traditionnalisme chinois), Taipei, Fengyun shidai, 1993. 2. Voir à ce sujet, Liu Xiaobo, Mori xingcunzhe de dubai (Monologue d'un rescapé du dernier jour), Taipei, Shibao wenhua chubanshe, Lishi yu xianchang congshu, 1992.

3. Paru dans Mingbao yuekan, juillet 1990, pp. 13-16.

4. Ah Q, personnage central de la nouvelle de Lu Xun, La véridique histoire d'Ah $Q$ (Ah $\mathrm{Q}$ zhengzhuan), qui personnifie depuis l'acceptation aveugle de tout avec la certitude d'être supérieur.

5. Paru dans Kaifang, novembre 1994.

6. Assoiffés (Kewang), série télévisée basée sur une nouvelle de Wang Shuo, et diffusée dans les années 1992-1993 avec un énorme succès.

7. La Capitale déchue, (Feidu), roman de Jia Pingwa, paru en 1993. Lire à ce sujet Annie Au-Yeung, "Un livre controversé", Perspectives chinoises $\mathrm{n}^{\circ} 21$, janvier-février 1994, p. 52.

8. Voir l'article de Jean-Philippe Béja, "Naissance d'un national-confucianisme", Perspectives chinoises n³0, juillet-août 1995, p. 6.

9. Empereurs des Qing, 1736-1796 et 1796-1821.

10. Vuir l'article de Chen Yan, "Fièvre du passé el crise identitaire", Perspectives chinoises $\mathrm{n}^{\circ} 28$, mars-avril 1995, p. 9.

\section{Perspectives Chinoises}

est disponible dans les librairies suivantes :

\section{PARIS}

Dunod Dauphine : Hall de l'Université, Place du Maréchal de Lattre de Tassigny, 75116 Paris.

Itinéraires : 60 rue Saint Honoré, 75001 Paris.

Fenêtre sur l'Asie : 49 rue Gay-Lussac, 75005 Paris.

Les FNAC : Montparnasse, Les Halles, La Défense, Etoile, Parly 2 et Vélizy 2.

L'Harmattan : 16 rue des Ecoles, 75005 Paris.

La Procure : 3 rue de Mézières, 75006 Paris.

Le Phénix : 72 boulevard de Sébastopol, 75003 Paris.

Librairie des sciences politiques : 30 rue St Guillaume, 75007 Paris.

You Feng : 45 rue Monsieur le Prince, 75006 Paris.

\section{HONG KONG}

Parenthèses : 4/F, Duke of Wellington House, 14 Wellington Street, Central. 